\title{
A new management strategy for renal angiomyolipomas: superselective arterial embolization in combination with radiofrequency ablation
}

\author{
Fengyong Liu, Hongjun Yuan, Xin Li, Jing Tang, Xiaomei Tian, Kan Ji \\ The First Medical Center of PLA General Hospital, Beijing 100853, China \\ Contributions: (I) Conception and design: F Liu; (II) Administrative support: F Liu; (III) Provision of study materials or patients: H Yuan; (IV) \\ Collection and assembly of data: X Li, J Tang; (V) Data analysis and interpretation: X Tian, K Ji; (VI) Manuscript writing: All authors; (VII) Final \\ approval of manuscript: All authors. \\ Correspondence to: Fengyong Liu. Department of Interventional Radiology, The First Medical Center of PLA General Hospital, 28 Fuxing Road, \\ Beijing 100853, China. Email: fengyongliu@aliyun.com.
}

Background: The present study analyzed the safety and efficacy of superselective arterial embolization (SAE) combined with radiofrequency ablation (RFA) to treat renal angiomyolipoma (RAML).

Methods: This retrospective study included the clinical data of 6 patients with RAML treated between March 2016 and October 2017. All patients underwent RFA treatment immediately after SAE guided by angio-computed tomography (angio-CT). The tumor size, renal function and complications, and time to recurrence were compared before and after treatment.

Results: The success rate of the SAE + RFA technique was $100 \%$, and no serious complications were found during or after surgery. After treatment, the patients' symptoms, including low back pain and hematuria, were relieved. The pre-treatment creatinine level $(55.6 \pm 11.0 \mu \mathrm{mol} / \mathrm{L})$ did not differ significantly from the post-treatment creatinine level $(68.2 \pm 13.7 \mu \mathrm{mol} / \mathrm{L})$, which was measured 1 day after surgery $(\mathrm{t}=1.75$, $\mathrm{P}=0.109)$. The mean follow-up time was $29.5 \pm 6.1$ months. By the end of the follow-up period, the maximum diameter of the tumor was $3.1 \pm 0.6 \mathrm{~cm}$, which was significantly smaller than the pre-treatment diameter of $5.9 \pm 1.01 \mathrm{~cm}(\mathrm{t}=5.83, \mathrm{P}<0.001)$. At 3 months after surgery, the maximum diameter of the tumor was reduced by $22-38 \%$ in all 6 patients. At the last follow-up examination, the maximum diameter of the tumor was reduced by $36-61 \%$. Contrast-enhanced CT or magnetic resonance imaging scans showed that the enhanced part of the original lesion had disappeared, the renal parenchyma was full of even fat signals, and only one patient had new enhanced lesions at the site of the original lesions.

Conclusions: SAE combined with RFA is a safe and effective treatment for RAML, and its strengths qualify it to become a new treatment strategy for RAML.

Keywords: Angiomyolipoma/therapy; embolization, therapeutic/methods; radiofrequency ablation (RFA); renal neoplasms/therapy

Submitted Oct 19, 2019. Accepted for publication Nov 07, 2019.

doi: $10.21037 /$ atm.2019.11.63

View this article at: http://dx.doi.org/10.21037/atm.2019.11.63

\section{Introduction}

Renal angiomyolipoma (RAML) is a benign tumor of the kidney that consists of atypical blood vessels, smooth muscle, and adipose tissue $(1,2)$. The treatment of RAML is related to tumor size and the presence of symptoms (3).
Regular clinical follow-up is available without special treatment for asymptomatic RAML $<4 \mathrm{~cm}$ in diameter, while partial nephrectomy or selective arterial embolization (SAE) is the preferred treatment for patients with RAML $\geq 4 \mathrm{~cm}$ in diameter and for those with symptomatic RAML $(4,5)$. According to the 2012 International Tuberous 
Sclerosis Complex Consensus Group surveillance and management recommendations (6), SAE is the first-line treatment for RAML causing acute bleeding. However, multiple postoperative complications, renal impairment, recurrence, and retreatment after embolization remain urgent problems. In recent years, the efficacy and safety of percutaneous ablation for RAML have been gradually confirmed $(1,7,8)$. The introduction and clinical application of angio-computed tomography (angio-CT) devices have enabled the integration of SAE and radiofrequency ablation (RFA), improved imaging quality, and made operations simpler and safer $(9,10)$. The present study retrospectively analyzed the results from 6 patients with RAML treated with SAE+RFA, to evaluate the feasibility, safety, and efficacy of this new combination approach.

\section{Methods}

\section{General information}

From March 2016 to October 2017, we implemented combined SAE and RFA treatment in 6 patients with RAML. The present study was undertaken in accordance with the Helsinki Declaration as revised in 2013, approved in advance by the hospital ethics committee, and informed consent was obtained from all participants prior to treatment. Among the 6 patients, 2 were male, and 4 were female. The ages ranged from 31 to 69 years, with a median age of 49 years. The tumor sizes ranged from 4.7 to $7.2 \mathrm{~cm}$, with an average of $5.9 \mathrm{~cm}$. The maximum diameters of the lesions were $6.3,7.0,5.2,4.7,7.2$, and $5.5 \mathrm{~cm}$. Two patients had low back pain, 1 had hematuria, and the other 3 were asymptomatic. Two patients had the lesion located in the renal hilum, 2 in the upper pole of the kidney, 1 in the middle of the kidney, and 1 in the inferior pole of the kidney. All patients underwent contrast-enhanced CT or contrast-enhanced magnetic resonance imaging (MRI) of the kidney before operation. All patients were diagnosed with renal hamartoma by 2 senior radiologists based on the typical imaging findings. The patients' routine blood parameters, liver and kidney function, and coagulation function were normal, and none of them had hypertension, myocarditis, or myocardial infarction.

\section{Devices and instruments}

Selective renal artery embolization and RFA were performed under the guidance of Angio-CT (SIEMENS
MIYABI), which was a combination of a Siemens Artis zee DSA machine and a Somatom Emotion 16-row spiral CT scanner (Siemens Medical Solutions AG, Erlangen, Germany). The RF device used was a US 1500 RITA RF system (Model 1500; RITA Medical System, Mountain View, CA, USA), and the RF needle was a RITA RF ablation electrode needle.

\section{Treatment strategy}

The Angio-CT consists of DSA and CT sharing a single treatment bed. SAE was performed under DSA guidance, and CT-guided RFA was carried out immediately after without moving the patient (Figure 1).

In step 1 (SAE), the patient was placed in the supine position, local anesthesia with $1 \%$ lidocaine was applied to facilitate a retrograde femoral artery puncture, and a $4 \mathrm{~F}$ sheath and a $4 \mathrm{~F}$ angiography catheter were placed. Angiography of the abdominal aorta and renal arteries was performed (Figure 1B) to identify the arteries supplying blood to the tumor, and a superselective microcatheter was advanced into the target vessel. After the proper positioning was confirmed by contrast injection, bleomycin-iodinated oil was slowly injected into the tumor nest (Figure 1C). Polyvinyl alcohol particles were used to consolidate embolization. For cases with a relatively large arteriovenous fistula or aneurysm, coil embolization was added. The treatment was terminated after angiography confirmed excellent deposition of the iodized oil or disappearance of the tumor staining.

Step 2 (RFA) took place immediately after chemoembolization. The DSA display screen was removed, and the C-arm of the DSA was moved toward the patient's foot to make room for the CT scan. After the CT scan, according to the tumor size, location, adjacent relationship, effect of intraoperative lipiodol deposition, and outcome of superselective embolization of the feeding artery during the SAE procedure, the imaging workstation was used to design and determine the target, path, angle, and depth of needle puncture for the RFA. Surface marking was then carried out. The puncture site was sterilized routinely, and $1 \%$ lidocaine was used for local anesthesia. The RFA electrode needle was then advanced until the needle tip reached the boundary of the RAML. A CT scan was carried out at least once during needle insertion to confirm the position of the needle tip. After the RFA needle reached the target point, another CT scan was taken to confirm successful placement of the RFA needle, and RFA was performed on the lesion 

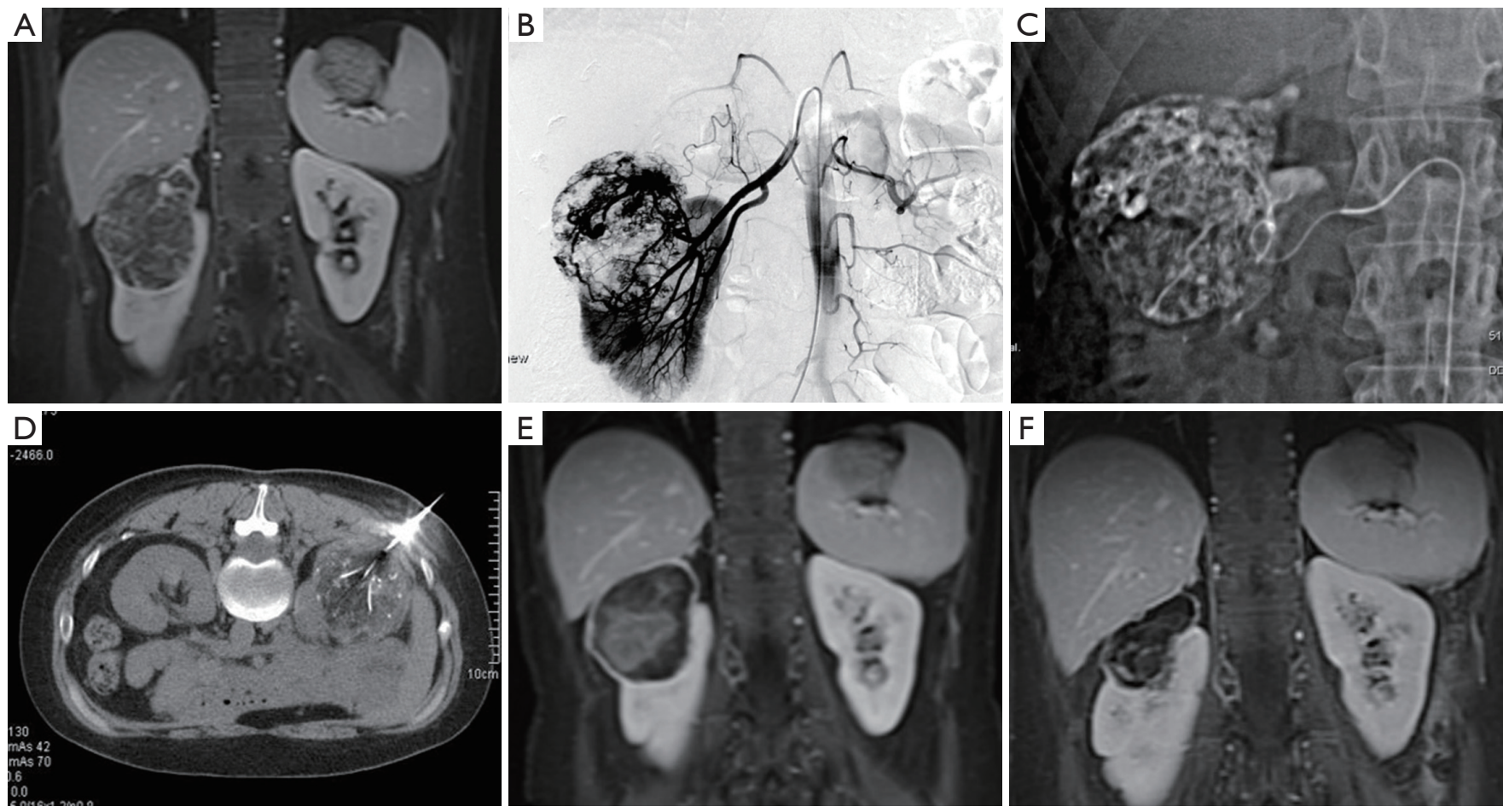

Figure 1 Patient 2, a 53-year-old female, underwent combined superselective arterial embolization (SAE) combined with radiofrequency ablation (RFA). (A) The patient was diagnosed with angiomyolipoma in the superior pole of the right kidney; (B) the digital subtraction angiography-guided angiography showed tumor staining; (C) SAE was performed; (D) computed tomography-guided radiofrequency ablation was carried out immediately after SAE; (E) contrast-enhanced magnetic resonance imaging (MRI), performed 3 months after surgery, showed that the enhanced part of the original lesion was reduced significantly, and mass-like uniform fat signals were seen in the lesion; (F) the contrast-enhanced MRI, performed 33 months after surgery, showed that the original lesion continued to shrink without enhancement, and patchy fat signals were seen in the lesion.

(Figure 1D). Real-time monitoring of the temperature and impedance of the lesion tissue was carried out, and the output power was adjusted according to the feedback. The treatment power ranged from 140-200 W. Overlapping ablation was performed to ensure complete ablation of the area surrounding the tumor. Ablation of each site lasted for 10-15 minutes. At the end of the ablation, the needle trajectory was ablated to reduce the risk of bleeding. Another CT scan was taken to observe the presence or absence of active bleeding and surrounding organ damage.

\section{Follow-up and efficacy evaluation}

Before surgery, the serum creatinine level and urea nitrogen level were measured, and a routine urine test was performed. A routine urine test was repeated each day after the operation. If findings were abnormal, the routine urine test was carried out continuously until the result became normal or other causes were identified. The serum creatinine level was measured on the $1^{\text {st }}$ and $3^{\text {rd }}$ day after surgery, and the results were compared with the preoperative level. Patients were followed up for 21-39 months. CT or MRI scan was performed every 3 months after surgery to detect any changes in the tumor and evaluate the therapeutic effect. According to the standardization of terminology and reporting criteria for image-guided tumor ablation (2009 and 2014) (11,12), efficacy was defined as the disappearance, degradation, and reduction of lesions or the lack of contrast-enhanced signs on contrast-enhanced CT scan or contrast-enhanced MRI scan. During the follow-up period, if the original lesion appeared to have a new blood supply, or a new nodule appeared close to the original lesion with confirmation by contrast-enhanced CT or MRI scan, the result was considered as local recurrence or residual tumor. 


\section{Statistical analysis}

SPSS 24.0 (IBM Corp., Armonk, NY, USA) was used for data analysis. The mean and median of the measured values were calculated and represented as mean \pm standard deviation $(\mathrm{x} \pm \mathrm{sd})$. The paired sample $t$-test was used for comparison, and $\mathrm{P}<0.05$ was considered to be statistically significant.

\section{Results}

\section{Success rate of surgery}

All 6 patients underwent successful treatment as planned. Both SAE and RFA procedures were performed by the same experienced doctor (FY Liu) and assistants, resulting in superselective cannulation into the tumor feeding arteries

Table 1 Mean and standard deviation (mean \pm SD) in changes in the serum creatinine level ${ }^{\dagger}(\mu \mathrm{mol} / \mathrm{L})$ before and after surgery

\begin{tabular}{lccc}
\hline Patients & Preoperative & $\begin{array}{c}\text { 1 day after } \\
\text { surgery }\end{array}$ & $\begin{array}{c}\text { 3 days after } \\
\text { surgery }\end{array}$ \\
\hline Patient 1 & 54 & 69 & 57 \\
Patient 2 & 62 & 72 & 54 \\
Patient 3 & 46 & 55 & 45 \\
Patient 4 & 49 & 61 & 45 \\
Patient 5 & 75 & 93 & 78 \\
Patient 6 & 48 & 59 & 42 \\
Mean \pm SD & $55.6 \pm 11.0$ & $68.2 \pm 13.7^{\star}$ & $53.5 \pm 13.3^{* *}$ \\
\hline${ }^{\dagger}$, the normal value of serum creatinine level is $62-115 \mu \mathrm{mmol} / \mathrm{L} ;$ \\
${ }^{*}, \mathrm{t}=1.75, \mathrm{P}=0.109 ;{ }^{* *}, \mathrm{t}=0.29, \mathrm{P}=0.772$.
\end{tabular}

and successful needle puncture into the target lesion during RFA. The success rate of both SAE and RFA procedures was $100 \%$.

\section{Intraoperative and postoperative complications}

Pain was the most common intraoperative side effect during the combined SAE and RFA procedure. In the 6 patients, 1 had mild pain, 4 had moderate pain, and 1 had severe pain. In the cases with severe intraoperative pain, morphine injection was given to relieve pain, and oxycodone hydrochloride tablets or morphine was given after surgery. Embolism/ablation syndrome developed in all 6 patients after surgery. The main symptoms were fever (below $38.5^{\circ} \mathrm{C}$ ), low back pain, fatigue, and general malaise. These symptoms were managed by appropriate symptomatic treatment. On the first postoperative day, the serum creatinine level increased slightly compared with the preoperative level to as high as about $27 \%$, which was still within the normal range, but practically returned to the preoperative level on the $3^{\text {rd }}$ day after surgery (Table 1). No serious complications such as ectopic embolism, renal abscess, or renal failure were observed.

\section{Postoperative imaging changes and outcomes}

The average follow-up duration was $29.5 \pm 6.12$ months. During the follow-up, the clinical symptoms caused by RAML were significantly reduced or disappeared (Table 2). The maximum diameter of the tumor before treatment was $4.7-7.2 \mathrm{~cm}$, with an average of $5.9 \pm 1.01 \mathrm{~cm}$, while the maximum diameter of the tumor at the last follow-up visit

Table 2 The length of follow-up (in months) and changes in maximum tumor diameter $(\mathrm{cm})$ before and after surgery

\begin{tabular}{|c|c|c|c|c|}
\hline Patients & $\begin{array}{l}\text { Follow-up duration } \\
\text { (months) }\end{array}$ & $\begin{array}{l}\text { Tumor before surgery } \\
\qquad(\mathrm{cm})\end{array}$ & $\begin{array}{c}\text { Tumor } 3 \text { months after } \\
\text { surgery }(\mathrm{cm})\end{array}$ & $\begin{array}{l}\text { Tumor at last follow-up } \\
(\mathrm{cm})\end{array}$ \\
\hline Patient 1 & 39 & 6.3 & $4.5(28 \%)$ & 3.6 (42\%) \\
\hline Patient 2 & 33 & 7.0 & $5.4(22 \%)$ & 3.8 (45\%) \\
\hline Patient 3 & 30 & 5.2 & 3.5 (32\%) & 2.4 (53\%) \\
\hline Patient 6 & 21 & 5.5 & $4.0(27 \%)$ & 3.5 (36\%) \\
\hline Total & $29.5 \pm 6.12$ & $5.9 \pm 1.01$ & $4.1 \pm 0.83^{\star}$ & $3.1 \pm 0.60^{\star \star}$ \\
\hline
\end{tabular}

The value in parentheses is the $\%$ of tumor shrinkage. * $t=1.81, P=0.100 ;{ }^{* \star}, t=5.83, P=0.000$. 

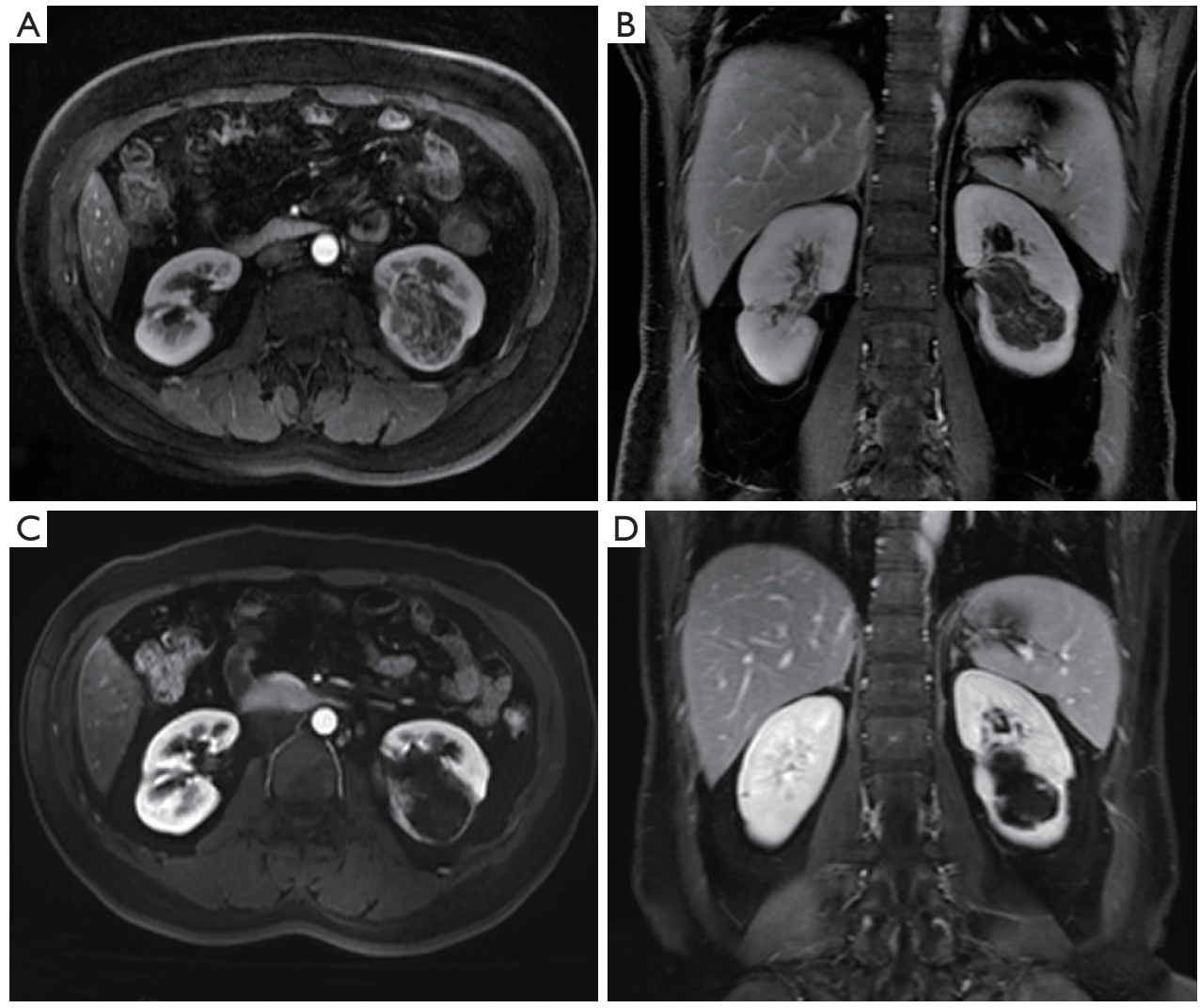

Figure 2 Patient 5, a 36-year-old female, underwent combined superselective arterial embolization (SAE) combined with radiofrequency ablation (RFA). (A,B) The patient was diagnosed with angiomyolipoma by MRI; (C,D) the contrast-enhanced MRI, performed 38 months after SAE + RFA, showed that the original lesion continued to shrink without enhancement.

was $3.1 \pm 0.60 \mathrm{~cm}$, which was significantly smaller than that before treatment $(t=5.83, \mathrm{p}<0.001)$. CT or MRI performed three months after the operation showed a $22-38 \%$ reduction in the maximum diameter of the tumor. The contrast-enhanced scan showed that the enhanced part of the original lesion disappeared, and the renal parenchyma was full of even fat signals. The maximum diameter of the tumor was reduced by $36-61 \%$ at the last follow-up. No enhanced lesions were observed, and 5 patients had decreased fat density. New enhanced lesions and fat tissues without enhancement were observed in 1 patient at the site of the original lesions (Figures 1,2).

\section{Discussion}

Due to the presence of immature vascular components, with the enlargement of the tumor, RAML may induce intratumoral aneurysms, subsequent intratumoral hemorrhage, or even tumor rupture, resulting in massive bleeding, and can thus endanger the lives of patients $(1,2,13)$. The probability of bleeding in RAML is positively correlated with tumor size, and as the tumor volume increases, the space-occupying effect causes a series of symptoms such as low back pain, hematuria, and inferior vena cava thrombosis $(14,15)$. De Luca et al. (5) recommended being on alert for spontaneous rupture of the tumor when the maximum diameter of the tumor is $>4 \mathrm{~cm}$. With the development of medical science and the improvement in people's living standards, the risk of bleeding and the space-occupying effect of RAML require a more prospective management strategy. Thus, we adopted the method of SAE+RFA (16).

SAE is a widely accepted intervention for the treatment of symptomatic RAML and a prophylactic treatment for asymptomatic RAML $>4 \mathrm{~cm}$ (17). However, some scholars (18) who investigated the clinical outcome of SAE treatment for symptomatic RAML found that the vascular tissue in the tumor is most sensitive to embolization, and 
the tumor shrinkage is mainly due to the large reduction in vascular components and smooth muscle atrophy. Because of the different proportions of blood vessels, smooth muscles, and fat in different tumors, the degree of tumor shrinkage after SAE treatment also differs; in particular, tumors with high fat content do not show significantly reduced volume $(19,20)$. We therefore decided to combine SAE therapy with RFA because our experience and published data $(1,17-20)$ suggest that treatment with SAE alone may not result in rapid tumor shrinkage. We did not apply SAE and RFA sequentially, but used both immediately, as sequential therapy may risk the disappearance of lipiodol or the granular embolic agent, the formation of newly-developed collateral vessels, and the recanalization of the embolized blood vessels. In addition, performing CT-guided RFA immediately after SAE is highly preferred among interventional doctors because of the gradual popularization of the application of DSA devices with CT function.

RFA is considered to be a minimally invasive treatment alternative to SAE and is effective for different sizes of RAML $(8,21)$. Matuszewski et al. (22) reported 2 cases of RAML with hematuria and low back pain as the main manifestations. The tumors were completely ablated after ultrasound-guided RFA, and no recurrence was observed at 1.5- and 3-year follow-up. Prevoo et al. (23) reported a patient with a solitary kidney in which the size of sporadic RAML was reduced from 4.5 to $2.9 \mathrm{~cm} 12$ months after RFA treatment, with no complications or RAML recurrence being observed at 12 months. Gregory et al. (24) reported 4 patients with RAML with an average diameter of $15.1 \mathrm{~cm}$, treated by CT-guided RFA. Two were treated with sequential RFA and SAE, with no tumor rupture or RAMLrelated symptoms occurring within 48 months of followup. Furthermore, percutaneous RFA treatment of RAML can maintain stable renal function and has relatively few complications (25). Castle et al. (7) used RFA to treat RAML and observed no intraoperative or postoperative complications. The creatinine level decreased to $0.03 \mathrm{mg} / \mathrm{dL}$ within 21 months of follow-up, and there were no transient hematuria or other symptoms. In the present study, we observed no serious complications during treatment. Intraoperative pain was managed by symptomatic treatment, and the patients tolerated the treatment successfully. The main postoperative complication was embolization/ablation syndrome (fever, pain, and other symptoms), with no significant fluctuations of serum creatinine levels. Thus, the feasibility and safety of immediate combined SAE and RFA treatment were affirmed.
Overall, we found the immediate combination of SAE and RFA to be safe and effective in the treatment of RAML. In the present study, the maximum diameter of the tumor before treatment was $4.7-7.2 \mathrm{~cm}$, with an average of $5.9 \pm 1.01 \mathrm{~cm}$, and the average duration of follow-up was $29.6 \pm 6.37$ months. The maximum diameter of the tumor at the last follow-up was $3.1 \pm 0.60 \mathrm{~cm}$, which was significantly smaller than the preoperative diameter $(\mathrm{t}=5.83, \mathrm{P}=0.000)$, representing an average reduction of about 47\% (36-61\%). Relative to the studies of Sooriakumaran et al. (26) and Castle et al. (7), in which the RFA technique was applied alone, our combination of SAE and RFA could reduce the tumor size more effectively without causing more complications. We speculate that this effect may be related to the synergistic effect of the immediate combination of SAE and RFA. Yuan et al. $(27,28)$ surmised that when the iodized oil is deposited well, immediate RFA can maximize the heat transfer of lipiodol, making the heat of the RFA needle quickly spread to the site in the lesion where the iodized oil is deposited, helping to inactivate the tumor. Especially for irregular tumor tissue, RFA alone cannot easily cover all three dimensions, while the angio-CT-guided instant combination of SAE and RFA can better transfer heat to the peripheral tumor tissue of RAML, reducing its recurrence. In addition, SAE blocks the arterial blood flow, reducing the risk of thermal deposition and bleeding during RFA ablation (29). We believe that SAE can be combined with RFA to maximize the synergistic effect of the two techniques.

We conclude that the immediate combination of SAE and RFA may be a safe and effective treatment strategy for RAML, which may reduce the tumor volume more quickly and reduce the potential bleeding risk compared to previous regimens. However, large-scale, multi-center, prospective studies are still needed to confirm this conclusion.

\section{Acknowledgments}

Funding: This work was supported by the National Natural Science Foundation of China (No. 81671800); Beijing Municipal Natural Science Foundation (7172204); Translational Medicine Project of PLA General Hospital (2017TM-013).

\section{Footnote}

Conflicts of Interest: The authors have no conflicts of interest to declare. 
Ethical Statement: The authors are accountable for all aspects of the work in ensuring that questions related to the accuracy or integrity of any part of the work are appropriately investigated and resolved. The study was approved in advance by the hospital ethics committee, and informed consent was obtained from all participants prior to treatment.

\section{References}

1. Flum AS, Hamoui N, Said MA, et al. Update on the Diagnosis and Management of Renal Angiomyolipoma. J Urol 2016;195:834-46.

2. Ge X, Xue X, Zhang G, et al. Successful treatment of liver and abdomen metastasis of renal epithelioid angiomyolipoma with apatinib: a case report. Transl Cancer Res 2017;6:1015-9.

3. Kuusk T, Biancari F, Lane B, et al. Treatment of renal angiomyolipoma: pooled analysis of individual patient data. BMC Urol 2015;15:123.

4. Wang C, Li X, Peng L, et al. An update on recent developments in rupture of renal angiomyolipoma. Medicine (Baltimore) 2018;97:e0497.

5. De Luca S, Terrone C, Rossetti SR. Management of renal angiomyolipoma: a report of 53 cases. BJU Int 1999;83:215-8.

6. Northrup H, Krueger DA. Tuberous sclerosis complex diagnostic criteria update: recommendations of the 2012 Iinternational Tuberous Sclerosis Complex Consensus Conference. Pediatr Neurol 2013;49:243-54.

7. Castle SM, Gorbatiy V, Ekwenna O, et al. Radiofrequency ablation (RFA) therapy for renal angiomyolipoma (AML): an alternative to angio-embolization and nephron-sparing surgery. BJU Int 2012;109:384-7.

8. Kiefer RM, Stavropoulos SW. The Role of Interventional Radiology Techniques in the Management of Renal Angiomyolipomas. Curr Urol Rep 2017;18:36.

9. Sone M, Arai Y, Sugawara S, et al. Angio-CT-Assisted Balloon Dissection: Protection of the Adjacent Intestine during Cryoablation for Patients with Renal Cancer. J Vasc Interv Radiol 2016;27:1414-9.

10. Piron L, Le Roy J, Cassinotto C, et al. Radiation Exposure During Transarterial Chemoembolization: Angio-CT Versus Cone-Beam CT. Cardiovasc Intervent Radiol 2019;42:1609-18.

11. Goldberg SN, Grassi CJ, Cardella JF, et al. Image-guided tumor ablation: standardization of terminology and reporting criteria. J Vasc Interv Radiol 2009;20:S377-90.
12. Ahmed M, Solbiati L, Brace CL, et al. Image-guided tumor ablation: standardization of terminology and reporting criteria—a 10-year update. Radiology 2014:273:241-60.

13. Shapiro DD, Abel EJ. Predicting aggressive behavior in small renal tumors prior to treatment. Ann Transl Med 2018;6:S132.

14. Yamakado K, Tanaka N, Nakagawa T, et al. Renal angiomyolipoma: relationships between tumor size, aneurysm formation, and rupture. Radiology 2002;225:78-82.

15. Fittschen A, Wendlik I, Oeztuerk S, et al. Prevalence of sporadic renal angiomyolipoma: a retrospective analysis of 61,389 in- and out-patients. Abdom Imaging 2014;39:1009-13.

16. Yuan H, Liu F, Li X, et al. Angio-CT-Guided Transarterial Chemoembolization Immediately in Combination with Radiofrequency Ablation for Large Hepatocellular Carcinoma. Acad Radiol 2019;26:224-31.

17. Murray TE, Doyle F, Lee M. Transarterial Embolization of Angiomyolipoma: A Systematic Review. J Urol 2015;194:635-9.

18. Hocquelet A, Cornelis F, Le Bras Y, et al. Longterm results of preventive embolization of renal angiomyolipomas: evaluation of predictive factors of volume decrease. Eur Radiol 2014;24:1785-93.

19. Kato H, Kuwatsuru R, Inoue T, et al. Superselective Transcatheter Arterial Embolization for Large Unruptured Renal Angiomyolipoma in Lymphangioleiomyomatosis. J Vasc Interv Radiol 2018;29:958-65.

20. Lin L, Li X, Guan H, et al. Renal function, complications, and outcomes of a reduction in tumor size after transarterial embolization for renal angiomyolipomas: a meta-analysis. J Int Med Res 2019;47:1417-28.

21. Fernández-Pello S, Hora M, Kuusk T, et al. Management of Sporadic Renal Angiomyolipomas: A Systematic Review of Available Evidence to Guide Recommendations from the European Association of Urology Renal Cell Carcinoma Guidelines Panel. Eur Urol Oncol 2019. [Epub ahead of print].

22. Matuszewski M, Michajłowski J, Bianek-Bodzak A, et al. Radiofrequency ablation of small symptomatic angiomyolipomas of the kidney: Report of two cases. Pol J Radiol 2010;75:68-71.

23. Prevoo W, van den Bosch MA, Horenblas S. Radiofrequency ablation for treatment of sporadic angiomyolipoma. Urology 2008;72:188-91.

24. Gregory SM, Anderson CJ, Patel U. Radiofrequency 
ablation of large renal angiomyolipoma: median-term follow-up. Cardiovasc Intervent Radiol 2013;36:682-9.

25. Sivalingam S, Nakada SY. Contemporary minimally invasive treatment options for renal angiomyolipomas. Curr Urol Rep 2013;14:147-53.

26. Sooriakumaran P, Gibbs P, Coughlin G, et al. Angiomyolipomata: challenges, solutions, and future prospects based on over 100 cases treated. BJU Int 2010;105:101-6.

27. Yuan H, Liu F, Li X, et al. Clinical efficacy of chemoembolization with simultaneous radiofrequency ablation for treatment of adrenal metastases from hepatocellular carcinoma. Cancer Imaging 2018;18:24.
28. Yuan H, Liu F, Li X, et al. Transcatheter arterial chemoembolization combined with simultaneous DynaCT-guided radiofrequency ablation in the treatment of solitary large hepatocellular carcinoma. Radiol Med 2019;124:1-7.

29. Sommer CM, Pallwein-Prettner L, Vollherbst DF, et al. Transarterial embolization (TAE) as add-on to percutaneous radiofrequency ablation (RFA) for the treatment of renal tumors: Review of the literature, overview of state-of-the-art embolization materials and further perspective of advanced image-guided tumor ablation. Eur J Radiol 2017;86:143-62.
Cite this article as: Liu F, Yuan H, Li X, Tang J, Tian X, Ji $\mathrm{K}$. A new management strategy for renal angiomyolipomas: superselective arterial embolization in combination with radiofrequency ablation. Ann Transl Med 2019;7(23):766. doi: 10.21037/atm.2019.11.63 